\title{
HUMAN ANGIOTENSIN-CONVERTING ENZYME 2 (ACE2) IS A RECEPTOR FOR HUMAN RESPIRATORY CORONAVIRUS NL63
}

\author{
M. K. Smith, Sonia Tusell, Emily A. Travanty, Ben Berkhout, \\ Lia van der Hoek, and Kathryn V. Holmes*
}

\section{INTRODUCTION}

Until 2003, only two human coronaviruses, HCoV-OC43 (group 2) and HCoV-229E (group 1), were well adapted to growth in tissue culture. These HCoVs have generally been associated with mild upper respiratory disease, although HCoV-229E can cause pneumonia in immunocompromised patients. ${ }^{5}$ In contrast, animal coronaviruses (CoVs) have been associated with a wide variety of diseases, many of them severe, in multiple animal and avian species. From the animal coronaviruses, a great deal about CoV biology and pathogenesis is known. The variety of animal hosts and diseases among CoVs are due in large part to differences in the spike glycoprotein. ${ }^{1,13,6,8,10} \mathrm{CoV}$ spikes are large type 1 membrane glycoproteins that are the major determinants of receptor specificity as well as virulence.

The recent discoveries of HCoV-NL63 (group 1) and its association with a variety of respiratory tract infections, including pneumonia and croup in young children, ${ }^{15}$ and HKU1 (group 2), isolated from a patient with pneumonia, ${ }^{16}$ shows that coronaviruses are emerging as important pathogens of the human lower respiratory tract.

The previously known group 1 CoVs use aminopeptidase N (APN) glycoprotein from their respective host species as their principal receptor. ${ }^{11}$ In addition, feline APN (fAPN) is a receptor for all of these group 1 coronaviruses (Table 1). ${ }^{12} \mathrm{HCoV}-\mathrm{NL} 63$ is most closely related to the group 1 coronaviruses. Indeed, the spike glycoprotein of HCoV-NL63 shares 55\% amino acid identity with the spike of HCoV-229E. We therefore tested whether this new virus would also utilize hAPN as its cellular receptor.

\footnotetext{
* M. K. Smith, Sonia Tusell, Emily A. Travanty, Kathryn V. Holmes, University of Colorado Health Sciences Center, Aurora, Colorado 80045. Ben Berkhout, Lia van der Hoek, University of Amsterdam, The Netherlands.
} 
Table 1. Group 1 coronavirus receptors. ${ }^{11}$

\begin{tabular}{|c|c|c|c|c|}
\hline Virus & hAPN $^{\mathrm{a}}$ & $\mathrm{pAPN}^{\mathrm{b}}$ & $\mathrm{cAPN}^{\mathrm{c}}$ & fAPN $^{d}$ \\
\hline $\mathrm{HCoV}-229 \mathrm{E}$ & + & - & - & + \\
\hline PRCoV & - & + & - & + \\
\hline TGEV & - & + & - & + \\
\hline $\mathrm{CCoV}$ & - & - & + & + \\
\hline $\mathrm{FCoV}$ & - & - & - & + \\
\hline
\end{tabular}

${ }^{\text {a }}$ Human aminopeptidase $\mathrm{N}$.

${ }^{\mathrm{b}}$ Porcine aminopeptidase $\mathrm{N}$.

${ }^{\mathrm{c}}$ Canine aminopeptidase $\mathrm{N}$.

${ }^{\mathrm{d}}$ Feline aminopeptidase $\mathrm{N}$.

\section{METHODS AND RESULTS}

\subsection{NL63 Infection of Cell Lines}

LLC-MK2 cells, Vero E6 cells, and a human lung fibroblast-like cell line, MRC-5 (ATCC \# CCL-171), were inoculated with NL63 and then observed for signs of CPE for 96 hours postinfection. Mild and transient CPE, consisting mostly of rounding and the appearance of vacuoles, was observed at 36 hours postinfection in all three cell types. HCoV-NL63 viral antigens were detected by immunofluorescence with a cross-reactive polyclonal goat antibody raised against $\mathrm{HCoV}-229 \mathrm{E}$ virions followed by fluorescein isothionate (FITC)-conjugated donkey anti-goat $\operatorname{IgG}$ as previously described. ${ }^{2}$ Viral antigen was detectable from 24 to 96 hours postinfection, with a maximum of about $25 \%$ of cells positive for antigen at 72 hours postinfection.

We inoculated LLC-MK2 and Vero E6 cells, which are permissive for HCoV-NL63 infection, ${ }^{14}$ as well as NIH-3T3 cells stably expressing hAPN (NIH-3T3/hAPN), BHK-21 cells stably expressing fAPN (BHK/fAPN), which are permissive for HCOV-229E infection, with either HCoV-NL63 or HCoV-229E at an MOI of 0.07. HCoV-229E and HCoV-NL63 viral antigens were both detected in susceptible cells with immunofluorescence. Viral antigens were detected in LLC-MK2 and Vero E6 cells inoculated with HCoV-NL63. No infection was detected in LLC-MK2 or Vero E6 cells inoculated with HCoV-229E. No viral antigens were detected in NIH-3T3/ hAPN or BHK/fAPN cells inoculated with HCoV-NL63, but robust infection was apparent in these cells after inoculation with $\mathrm{HCoV}-229 \mathrm{E}$ (Table 2).

The observations that both SARS-CoV and HCoV-NL63 grow in LLC-MK2 and Vero E6 cells led us to test the hypothesis that HCoV-NL63 uses the same receptor as SARS-CoV, angiotensin-converting enzyme 2 (ACE2). ${ }^{9}$ BHK-21 cells were transiently transfected with a plasmid encoding full-length human ACE2 (hACE2, kindly provided by Michael Farzan), and inoculated 48 hours after transfection with HCoV-NL63 or HCoV-229E. HCoV-NL63 antigen was detected in hACE2-transfected BHK-21 cells, but not in non-transfected controls. No HCoV-229E antigen was detected in either transfected or hACE2-negative control BHK-21 cells. ACE2-transfected cells were permissive for HCoV-NL63 replication, with peak yields of $10^{5} \mathrm{TCID}_{50} / \mathrm{ml}$ as assayed on LLC-MK2 cells.

To determine which receptor HCoV-NL63 uses to infect MRC-5 cells, we tested MRC-5s for surface expression of hACE2 by flow cytometry. MRC-5 cells expressed a small amount of ACE2, relative to LLC-MK2 and Vero E6 cells (Table 3). 
Table 2. Susceptibility of cultured cells to HCoV-NL63 and HCoV-229E infection.

\begin{tabular}{lcc}
\multicolumn{3}{c}{ Virus } \\
\hline Cell type & HCoV-229E & $\frac{\text { HCoV-NL63 }}{+}$ \\
LLC-MK2 & - & + \\
VERO E6 & - & + \\
MRC-5 & + & - \\
BHK & - & + \\
BHK/hACE2 & - & - \\
BHK/fAPN & + & + \\
\hline
\end{tabular}

\section{DISCUSSION}

In this paper, we show that infectious HCoV-NL63 used human ACE2 as an efficient receptor and that this group 1 coronavirus cannot use feline APN, the receptor for all other group 1 coronaviruses tested, or human APN, the principal receptor for $\mathrm{HCoV}$ 229E. Hoffmann et al., also showed that human ACE2 served as a receptor for HCoVNL63 using retrovirus pseudotyped with HCoV-NL63 spike glycoprotein. ${ }^{4}$

The finding that HCoV-NL63 uses ACE2 as a receptor is surprising in that HCoVNL63 is most closely related to $\mathrm{HCoV}-229 \mathrm{E}$, and its genome structure is most similar to that of PEDV in group 1, while SARS-CoV, which also uses human ACE2 as a receptor, is a distantly related member of group 2 coronaviruses. Although the group 1 spikes studied to date share no more than $48 \%$ amino acid sequence identity, all but HCoVNL63 are able to use fAPN as a receptor.

Interestingly, although the spike glycoprotein of HCoV-NL63 shares only $25 \%$ amino acid sequence identity with that of SARS-CoV (Urbani strain), the two viruses use the same receptor. It is possible that the two viruses have evolved independently to bind to ACE2. The finding that HCoV-NL63 and SARS-CoV utilize the same human receptor glycoprotein has important implications for coronavirus evolution. The genomes of all group 2 coronaviruses except SARS-CoV contain a gene encoding an HE glycoprotein that binds to $\mathrm{O}$-acetylated sialic acids, ${ }^{7}$ apparently derived by recombination of the mRNA encoding $\mathrm{HE}$ from influenza $\mathrm{C}$ with the genome of an ancestral group 2 coronavirus. ${ }^{7}$ Group 1 coronaviruses including HCoV-NL63 lack this HE gene. Perhaps both HCoV-NL63 and SARS-CoV descended from a common ancestral coronavirus that used ACE2 as its receptor, and this lineage split from the ancestral group 2 coronaviruses before the HE gene was acquired.

Understanding HCoV-NL63 S glycoprotein and how its interactions with its receptor, hACE2, will elicudate how pathogenic coronaviruses emerge and may suggest new strategies for prevention and therapy.

Table 3. Expression of ACE2 on Vero E6, LLC-MK2, and MRC-5 cells.

\begin{tabular}{lcc}
\multicolumn{2}{c}{ Percent cells gated } \\
\hline Cell type & Control antibody & Anti-ACE2 \\
\cline { 2 - 3 } Vero E6 & 3.1 & 77.6 \\
LLC-MK2 & 17.7 & 70.8 \\
MRC-5 & 7.7 & 27.0 \\
\hline
\end{tabular}




\section{ACKNOWLEDGMENTS}

The authors are grateful for helpful discussions with D. Wentworth, T. Miura, S. Jeffers, L. Thackray, and B. Turner. This research was supported by NIH grant RO1 A1 26075.

\section{REFERENCES}

1. M. L. Ballesteros, C. M. Sanchez, and L. Enjuanes, Two amino acid changes at the N-terminus of transmissible gastroenteritis coronavirus spike protein result in the loss of enteric tropism, Virology 227 378-388 (1997).

2. A. Bonavia, B. D. Zelus, D. E. Wentworth, P. J. Talbot, and K. V. Holmes, 3 A.D. Identification of a receptor-binding domain of the spike glycoprotein of human coronavirus HCoV-229E, J. Virol. 77, 2530$2538(2003)$

3. R. A. Fouchier, N. G. Hartwig, T. M. Bestebroer, B. Niemeyer, J. C. De Jong, J. H. Simon, and A. D. Osterhaus, A previously undescribed coronavirus associated with respiratory disease in humans, Proc. Natl. Acad. Sci. USA 101, 6212-6216 (2004).

4. H. Hofmann, K. Pyrc, L. van der Hoek, M. Geier, B. Berkhout, and S. Pohlmann, Human coronavirus NL63 employs the severe acute respiratory syndrome coronavirus receptor for cellular entry, Proc. Natl. Acad. Sci. USA 102, 7988-7993 (2005).

5. F. Pene, A. Merlat, A. Vabret, F. Rozenberg, A. Buzyn, F. Dreyfus, A. Cariou, F. Freymuth, and P. Lebon, Coronavirus 229E-related pneumonia in immunocompromised patients, Clin. Infect. Dis. 37, 929-932 (2003).

6. J. D. Rempel, S. J. Murray, J. Meisner, and M. J. Buchmeier, Mouse hepatitis virus neurovirulence: evidence of a linkage between $\mathrm{S}$ glycoprotein expression and immunopathology, Virology 318, 45-54 (2004).

7. S. L. Smits, G. J. Gerwig, A. L. van Vliet, et al., Nidovirus sialate-O-acetylesterases: evolution and substrate specificity of coronaviral and toroviral receptor-destroying enzymes, J. Biol. Chem. 280, 6933-6941 (2005).

8. H. D. Song, C. C. Tu, G. W. Zhang, et al., Cross-host evolution of severe acute respiratory syndrome coronavirus in palm civet and human, Proc. Natl. Acad. Sci. USA 102, 2430-2435 (2005).

9. J. Sui, W. Li, A. Murakami, et al., Potent neutralization of severe acute respiratory syndrome (SARS) coronavirus by a human $\mathrm{mAb}$ to $\mathrm{S} 1$ protein that blocks receptor association, Proc. Natl. Acad. Sci. USA 101, 2536-2541 (2004).

10. L. B. Thackray and K. V. Holmes, Amino acid substitutions and an insertion in the spike glycoprotein extend the host range of the murine coronavirus MHV-A59, Virology 324, 510-524 (2004).

11. D. B. Tresnan and K. V. Holmes, Feline aminopeptidase N is a receptor for all group I coronaviruses, $A d v$ Exp. Med. Biol. 440, 69-75 (1998).

12. D. B. Tresnan, R. Levis, and K. V. Holmes, Feline aminopeptidase N serves as a receptor for feline, canine, porcine, and human coronaviruses in serogroup I, J. Virol. 70, 8669-8674 (1996).

13. J. C. Tsai, L. de Groot, J. D. Pinon, K. T. Iacono, J. J. Phillips, S. H. Seo, E. Lavi, and S. R. Weiss, Amino acid substitutions within the heptad repeat domain 1 of murine coronavirus spike protein restrict viral antigen spread in the central nervous system, Virology 312, 369-380 (2003).

14. L. van der Hoek, K. Pyrc, M. F. Jebbink, et al., Identification of a new human coronavirus, Nat. Med. 10, 368-373 (2004).

15. L. van der Hoek, K. Sure, G. Ihorst, et al., Croup is associated with the novel coronavirus NL63, PLoS. Med. 2, e240 (2005).

16. P. C. Woo, S. K. Lau, C. M. Chu, et al., Characterization and complete genome sequence of a novel coronavirus, coronavirus HKU1, from patients with pneumonia, J. Virol. 79, 884-895 (2005). 